\title{
NOTES ON QUESTIONS OF W. VOGEL CONCERNING THE CONVERSE TO BÉZOUT'S THEOREM
}

\author{
by THILO PRUSCHKE
}

(Received 11th November 1991)

\begin{abstract}
Lazarsfeld proved a bound for the excess dimension of an intersection of irreducible and reduced schemes. Flenner and Vogel gave another approach for reduced, non-degenerate schemes which are connected in codimension one, using the intersection algorithm of Stückrad and Vogel and defining a new multiplicity $k$. Renschuch and Vogel considered a condition to ensure that there is no degeneration for more than two schemes. We define an integer which enables us to unify these methods. This allows us to generalize the result of Flenner and Vogel to non-reduced schemes by comparing the multiplicities $j$ and $k$. Using this point of view we give applications to converses of Bézout's theorem; in particular we investigate the Cohen-Macaulay case.
\end{abstract}

1991 Mathematics subject classification: Primary 14C17. Secondary $13 \mathrm{H} 15$.

H. Flenner and W. Vogel gave a first approach to the converse to Bézout's theorem in [1]. Since then there has been other work on this subject (see $[2,9,13,14]$ ). The converse to Bézout's theorem consists of an answer to the following question: under which conditions does the situation of a proper intersection follow from a Bézout'sequality? In this connection, in $[13,14]$ a relation between the excess dimension and dimensions of vector spaces of linear forms is considered. We want to investigate this connection rather more deeply.

We always consider the intersection of two or more than two schemes. This transition from the case of two schemes is not quite trivial, as the discussion of example 12.3 .5 of [3, p. 225] in [9, p. 316, Remark 1] shows. In [3, p. 225], one assumption is missed and this is corrected by an unnecessarily strong condition. This condition is commented on and also weakened in [4]. We give here a new condition.

In particular, in Proposition 2.4 and Theorem 2.9., we give generalizations of Theorem 4.2 of [1]. These generalizations are based on Proposition 2.2 and Lemma 2.8 . In the latter we compare the intersection multiplicities $j$ (see $[3,11,12])$ and $k$ (see [1], where the notation $\tilde{j}$ was used). This allows us to pass from the intersection of reduced schemes to that of general pure dimensional schemes. The main result of $H$. Flenner and W. Vogel in [1] and the result of L. O'Carroll in [8] treat the reduced case. Theorem 2.9 especially has some interesting applications. For example, we use this result in Section 3 to give a new proof of Proposition 3.12 of [2] and to study the CohenMacaulay case. Also, we give in Corollary 3.3 a partial answer to the question following Theorem 7 in [14]. We generalize the Theorem of [13] in the geometrically more 
interested case by considering all irreducible (i.e. idealtheoretically isolated) components and not only the highest dimensional components (see Theorem 2.7). (The former are the geometrically visible components.)

\section{Notation and preliminary results}

Let us fix the following notation for the whole paper. Let $K$ be an algebraically closed field and $X_{1}, \ldots, X_{r}(r \geqq 2)$ pure dimensional subschemes of $\mathbb{P}_{K}^{n}$ with defining homogeneous ideals $\alpha_{1}, \ldots, \alpha_{r}$ in $R_{0}:=K\left[x_{0}, \ldots, x_{n}\right]$. We introduce $r$ copies $R_{i}:=$ $K\left[x_{i 0}, \ldots, x_{i r}\right], 1 \leqq i \leqq r$, of $R_{0}$ and denote by $\alpha_{i}^{\prime}$ the corresponding ideal of $\alpha_{i}$ in $R_{i}$.

We set

$$
\begin{aligned}
& R:=K\left[x_{i j} ; 1 \leqq i \leqq r, 0 \leqq j \leqq n\right], \\
& c:=\text { the diagonal ideal in } R, \text { which is generated by }\left\{x_{1 j}-x_{i j} ; 2 \leqq i \leqq r, 0 \leqq j \leqq n\right\}, \text { and } \\
& m:=(r-1) \cdot(n+1)-1 .
\end{aligned}
$$

The join variety is given by $J=\left(\alpha_{1}^{\prime}+\cdots+\alpha_{r}^{\prime}\right) \cdot R$ in $\mathbb{P}_{K}^{m}$. Furthermore, we introduce new independent variables $u_{k i j}$ over $K$, for $0 \leqq k \leqq m, 1 \leqq i \leqq r$ and $0 \leqq j \leqq n$. Let $\bar{K}$ denote the algebraic closure of

$$
K\left(u_{k i j} ; 0 \leqq k \leqq m, 1 \leqq i \leqq r, 0 \leqq j \leqq n\right)
$$

and we set

$$
\bar{R}:=\bar{K}\left[x_{i j} ; 1 \leqq i \leqq r, 0 \leqq j \leqq n\right] .
$$

In this ring we consider so-called generic linear forms $l_{0}, \ldots l_{m}$, with

$$
l_{k}:=\sum_{\substack{2 \leq i \leq r \\ 0 \leqq j \leqq n}} u_{k i j} \cdot\left(x_{1 j}-x_{i j}\right), \text { for } k=0, \ldots, m
$$

It is clear that $c \cdot \bar{R}=\left(l_{0}, \ldots, l_{m}\right)$. We set

$$
\begin{aligned}
& \delta:=\operatorname{Krull}-\operatorname{dim} J \cdot \bar{R}=\sum_{i=1}^{r} \operatorname{dim} X_{i}+(r-1), \\
& d:=\operatorname{Krull}-\operatorname{dim}\left(\alpha_{1}+\cdots+\alpha_{r}\right)=\operatorname{dim}\left(X_{1} \cap \cdots \cap X_{r}\right)+1 \text {, and } \\
& e\left(X_{1}, \ldots, X_{r}\right):=\operatorname{dim}\left(X_{1} \cap \cdots \cap X_{r}\right)-\sum_{i=1}^{r} \operatorname{dim} X_{i}+n \cdot(r-1) .
\end{aligned}
$$

The latter is called the excess dimension of $X_{1}, \ldots, X_{r}$, and we often write it simply as $e$. 
In the following, we describe the intersection algorithm of $[11,12]$. So we define the sequence $(J \cdot \bar{R})_{i}$, for $i=1, \ldots, \delta-d-1=m-e$, by

$$
\begin{gathered}
(J \cdot \bar{R})_{-1}:=J \cdot \bar{R} \\
(J \cdot \bar{R})_{k}:=l_{k} \cdot \bar{R}+U\left((J \cdot \bar{R})_{k-1}\right), \text { for } k=0, \ldots, m-e,
\end{gathered}
$$

where $U(\ldots)$ is the intersection of all highest dimensional primary ideas belonging to (...).

Furthermore, we set

$$
\begin{gathered}
\mathfrak{U}_{0}:=U\left((J \cdot \bar{R})_{m-e}\right), \\
\mathfrak{U}_{s}:=\cap q \text { for } s=1, \ldots, e-1,
\end{gathered}
$$

where the intersection is taken over all primary ideals $q$ belonging to

$$
U\left(\mathfrak{U}_{s-1}+l_{m-e+s-1}\right)
$$

such that $c \cdot \bar{R}$ is not contained in the associated prime of $q$. Now we describe the construction of the collection $\mathscr{C}\left(X_{1}, \ldots, X_{r}\right)$ (in short, $\left.\mathscr{C}\right)$ and define the intersection multiplicities $j\left(X_{1}, \ldots, X_{r} ; C\right)$ and $k\left(X_{1}, \ldots, X_{r} ; C\right)$.

The collection $\mathscr{C}$ contains irreducible, reduced schemes $C$ of $\mathbb{P}_{K}^{n}$, which satisfy

$$
\operatorname{dim}\left(X_{1} \cap \cdots \cap X_{r}\right) \geqq \operatorname{dim} C \geqq \sum_{i=1}^{r} \operatorname{dim} X_{i}-(r-1) \cdot n
$$

To define the elements of $\mathscr{C}$ of dimension

$$
\operatorname{dim}\left(X_{1} \cap \cdots \cap X_{r}\right)-i, \text { for } i=0, \ldots, e,
$$

we decompose

$$
U\left(\mathfrak{U}_{i}+l_{m-e+i} \cdot \bar{R}\right)
$$

in the following way.

$$
U\left(\mathfrak{U}_{i}+l_{m-e+i} \cdot \bar{R}\right)=\cap q \cap \mathfrak{U}_{i+1}
$$

where the first intersection is taken over all primary ideals $q$ belonging to $U\left(\mathfrak{U}_{i}+l_{m-e+i} \cdot \bar{R}\right)$, where associated primes $p$ contain the diagonal ideal $c \cdot \bar{R}$. These prime ideals modulo $c \cdot \bar{R}$ define irreducible and reduced subschemes of $X_{1} \cap \cdots \cap X_{\text {, in }}$ $\mathbb{P}_{\bar{K}}^{n}$ - the elements of the collection $\mathscr{C}$. 
Such a $C$ is defined by the prime ideal $p:=I(C)$ in $\bar{K}\left[x_{0}, \ldots, x_{n}\right]$. The intersection numbers of $X_{1}, \ldots, X_{r}$ along $C$, with $C$ having $\operatorname{dimension} \operatorname{dim}\left(X_{1} \cap \cdots \cap X_{r}\right)-i$, are given by

$$
\begin{gathered}
j\left(X_{1}, \ldots, X_{r} ; C\right):=\text { length of } U\left(\mathfrak{U}_{i}+l_{m-e+i} \cdot \bar{R}\right)_{(p+c) \cdot \bar{R}}, \text { and } \\
k\left(X_{1}, \ldots, X_{r} ; C\right):=\text { length of }\left(U\left(\mathfrak{U}_{i}+l_{m-e+i} \cdot \bar{R}\right)+c \cdot \bar{R}\right)_{(p+c) \cdot \bar{R}}
\end{gathered}
$$

It is clear that $j\left(X_{1}, \ldots, X_{r} ; C\right) \geqq k\left(X_{1}, \ldots, X_{r} ; C\right)$ for all $C \in \mathscr{C}$. We also use $\mathscr{C}_{\text {irr }}\left(X_{1}, \ldots, X_{r}\right)$ (or $\mathscr{C}_{\text {irr }}$ ) to denote the set of all irreducible components of $X_{1} \cap \cdots \cap X_{r}$ and $\mathscr{C}_{h}\left(X_{1}, \ldots, X_{r}\right)$ (or $\left.\mathscr{C}_{h}\right)$ to denote the set of the highest dimensional components of $\mathscr{C}$. (These are the components $C$ with $\operatorname{dim} C=\operatorname{dim}\left(X_{1} \cap \cdots \cap X_{r}\right)$ ). Then we get the following inclusions:

$$
\mathscr{C}_{h} \subseteq \mathscr{C}_{\text {irr }} \subseteq \mathscr{C}
$$

Now we state the main results from $[11,12]$ and $[1]$.

Lemma 1.1. Let $X_{1}, \ldots, X_{r}$ be pure dimensional subschemes in $\mathbb{P}_{K}^{n}$. Then

$$
\prod_{i=1}^{r} \operatorname{deg} X_{i}=\sum_{C=\mathscr{C}} j\left(X_{1}, \ldots, X_{r} ; C\right) \cdot \operatorname{deg} C .
$$

Definition 1. The homogeneous ideals $\alpha_{1}, \ldots, \alpha_{r} \subseteq K\left[x_{0}, \ldots, x_{n}\right]$ satisfy the (*)condition if no linear form is contained in

$$
\alpha_{i} \cap\left(\alpha_{1}+\cdots+\alpha_{i-1}+\alpha_{i+1}+\cdots+\alpha_{r}\right), \text { for } i=1, \ldots, r
$$

This definition enables us to state the main result from [1].

Lemma 1.2. Let $X_{1}, \ldots, X_{r}$ be pure dimensional and reduced subschemes in $\mathbb{P}_{K}^{n}$ which are connected in codimension one. If the defining homogeneous ideals in $K\left[x_{0}, \ldots, x_{n}\right]$ satisfy the (*)-condition, then

$$
\prod_{i=1}^{r} \operatorname{deg} X_{i} \geqq \sum_{C \in \mathscr{C}_{\text {irr }}} k\left(X_{1}, \ldots, X_{r} ; C\right) \cdot \operatorname{deg} C+e .
$$

(A scheme $X$ is connected in codimension one if for any closed subscheme $Y$ of $X$ with $\operatorname{codim}(Y, X)>1$ the set $X \backslash Y$ is connected. For applications of connectedness in codimension one, see $[7,10]$.)

We need also a bilinear property of the above intersection algorithm involving the join construction in $\mathbb{P}_{\bar{K}}^{n}$. This is given by the following so-called theorem of additivity and reduction (see for instance $[5,13]$ ). 
Lemma 1.3. Let $X_{1}, \ldots, X_{r}$ be pure dimensional subschemes in $\mathbb{P}_{K}^{n}$ with defining ideals $\alpha_{1}, \ldots, \alpha_{r} \subseteq K\left[x_{0}, \ldots, x_{n}\right]$. We consider the primary decomposition of $\alpha_{i}=q_{1}^{(i)} \cap \cdots \cap q_{s_{i}}^{(i)}$, and put $p_{j}^{(i)}$ to be the prime ideals belonging to $q_{j}^{(i)}$. Furthermore, let $X_{i j}$ (respectively $V_{i j}$ ) be the subscheme of $\mathbb{P}_{K}^{n}$ defined by $q_{j}^{(i)}\left(\right.$ respectively $\left.p_{j}^{(i)}\right)$ and let $1_{i j}:=$ length of $q_{j}^{(i)}$. Then

(i) $\mathscr{C}\left(X_{1}, \ldots, X_{r}\right)=\bigcup \mathscr{C}\left(X_{1 j_{1}}, \ldots, X_{r j_{r}}\right)=\bigcup \mathscr{C}\left(V_{1 j_{1}}, \ldots, V_{r j_{r}}\right)$, where the union is taken over all $1 \leqq j_{i} \leqq s_{i}, 1 \leqq i \leqq r$,

(ii) $j\left(X_{1}, \ldots, X_{r} ; C\right)=\sum 1_{1 j_{1}} \cdot \ldots \cdot 1_{r j_{r}} \cdot j\left(V_{1 j_{1}}, \ldots, V_{r j_{r}} ; C\right)$, where the sum is taken over all $1 \leqq j_{i} \leqq s_{i}, 1 \leqq i \leqq r$, where we set $j\left(V_{1 j_{1}}, \ldots, V_{r j_{r}} ; C\right)=0$ for $C \notin \mathscr{C}\left(V_{1 j_{1}}, \ldots, V_{r j_{r}}\right)$.

We now give definitions for commonly used abbreviations.

Let $X$ be a pure dimensional scheme in $\mathbb{P}_{K}^{n}$ with defining homogeneous ideal $\alpha \subseteq R:=K\left[x_{0}, \ldots, x_{n}\right]$. Then

$$
X_{\text {red }} \text { is the scheme which is given by } \operatorname{Rad}(\alpha) \text {. }
$$

$\bar{X}$ is the scheme which is given by the ideal $\left([\alpha]_{1}\right)$ which is generated by the linear forms of $\alpha$.

Let $\alpha$ be an arbitrary homogeneous ideal of $R$. Then the volume function $V(\cdot, \alpha): \mathbb{Z} \rightarrow \mathbb{Z}$ is defined as follows

$$
V(t, \alpha):=\operatorname{dim}_{K}[\alpha]_{t}
$$

where $[\alpha]_{t}$ denotes the vector space over $K$ of all forms of $\alpha$, which have the degree $t$. (For the basic properties of the volume function see [6].)

Let $X_{1}, \ldots, X_{r}$ be pure dimensional schemes in $\mathbb{P}_{K}^{n}$. Then we put the excess dimension $e$ by setting

$$
\begin{aligned}
& e:=e\left(X_{1}, \ldots, X_{r}\right) \\
& :=\operatorname{dim}\left(X_{1} \cap \cdots \cap X_{r}\right)-\sum_{i=1}^{r} \operatorname{dim} X_{i}+n \cdot(r-1) .
\end{aligned}
$$

Let $Z$ be a closed subscheme of $\mathbb{P}_{K}^{n}$. Then we set

$$
\operatorname{deg} Z:=\sum_{c} \lg \left(\mathcal{O}_{z, c}\right) \cdot \operatorname{deg} C,
$$

where the sum is taken over all irreducible components $C$ of $Z$, and we put $\lg \left(\mathcal{O}_{Z, C}\right)=$ length of $\mathcal{O}_{Z, C}$.

\section{Converses to Bézout's theorem}

In this section we give some converses to Bézout's theorem. The main points are the 
decomposition into irreducible and reduced components using Lemma 1.3 and an estimation of the difference of the two multiplicities $j-k$. This allows us to consider the intersection of general schemes rather than just reduced schemes. First we give the definition of the number $\mathrm{rg}$ and apply it to the converse to Bézout's theorem.

Definition 2. Let $X_{1}, \ldots, X_{r}$ be pure dimensional subschemes in $\mathbb{P}_{K}^{n}$ with defining homogeneous ideals $\alpha_{1}, \ldots, \alpha_{r} \subseteq K\left(x_{0}, \ldots, x_{n}\right]$. Then we put

$$
\begin{aligned}
\mathrm{rg} & :=\operatorname{rg}\left(X_{1}, \ldots, X_{r}\right):=\operatorname{rg}\left(\alpha_{1}, \ldots, \alpha_{r}\right) \\
& :=\sum_{i=1}^{r} V\left(1, \alpha_{i}\right)-V\left(1, \sum_{i=1}^{r} \alpha_{i}\right), \\
\mathrm{rg}_{\mathrm{red}}: & =\operatorname{rg}\left(\left(X_{1}\right)_{\mathrm{red}}, \ldots,\left(X_{r}\right)_{\mathrm{red}}\right) .
\end{aligned}
$$

We obtain also by simple computation that

$$
\operatorname{rg}\left(X_{1}, \ldots, X_{r}\right)=e\left(\bar{X}_{1}, \ldots, \bar{X}_{r}\right)
$$

Proposition 2.1. Let $\alpha_{1}, \ldots, \alpha_{r}$ be unmixed homogeneous ideals of $R:=K\left[x_{0}, \ldots, x_{n}\right]$. Then these ideals satisfy the $\left(^{*}\right)$-condition if and only if $\operatorname{rg}\left(\alpha_{1}, \ldots, \alpha_{r}\right)=0$.

Proof. We compute $V\left(1, \sum_{i=1}^{r} \alpha_{i}\right)$ using the dimension theorem for vector spaces.

$$
\begin{aligned}
V\left(1, \sum_{i=1}^{r} \alpha_{i}\right) & =V\left(1, \alpha_{i}\right)+V\left(1, \sum_{\substack{j=1 \\
j \neq i}}^{r} \alpha_{j}\right)-V\left(1, \alpha_{i} \cap \sum_{\substack{j=1 \\
j \neq i}}^{r} \alpha_{j}\right) \\
& =\sum_{i=1}^{r} V\left(1, \alpha_{i}\right)-V\left(1, \alpha_{i} \cap \sum_{\substack{j=1 \\
j \neq i}}^{r} \alpha_{j}\right) \\
& -\sum_{\substack{k=1 \\
k \neq i}}^{r-1} V\left(1, \alpha_{k} \cap \sum_{\substack{j=k+1 \\
j \neq i}}^{r} \alpha_{j}\right)
\end{aligned}
$$

for an arbitrary $i(1 \leqq i \leqq r)$.

If the $\left(^{*}\right)$-condition is satisfied, then the latter two terms vanish, and we get

$$
\sum_{i=1}^{r} V\left(1, \alpha_{i}\right)-V\left(1, \sum_{i=1}^{r} \alpha_{i}\right)=0
$$


On the other hand, if formula (2) holds, then certainly the second term in (1) vanishes, i.e. the $\left(^{*}\right)$-condition holds.

Proposition 2.2. Let $X_{1}, \ldots, X_{r}$ be pure dimensional subschemes of $\mathbb{P}_{K}^{n}$ with defining homogeneous ideal $\alpha_{1}, \ldots, \alpha_{r}$ in $K\left[x_{0}, \ldots, x_{n}\right]$. Then

(i) $\operatorname{rg}\left(\alpha_{1}, \ldots, \alpha_{r}\right)=V(1, J \cdot R \cap c \cdot R)$,

(ii) $\operatorname{rg}\left(\alpha_{1}, \ldots, \alpha_{r}\right) \leqq e\left(X_{1}, \ldots, X_{r}\right)$.

The following conditions

(iii) $\operatorname{rg}\left(\alpha_{1}, \ldots, \alpha_{r}\right)=e\left(X_{1}, \ldots, X_{r}\right)$,

(iv) $l_{m-e+1}, \ldots, l_{m} \in\left(J, l_{0}, \ldots, l_{m-e}\right) \cdot \bar{R}$,

(v) $\prod_{i=1}^{r} \operatorname{deg} X_{i}=\sum_{C \in \mathbb{C}}^{m} k\left(X_{1}, \ldots, X_{r} ; C\right) \cdot \operatorname{deg} C$, where $\mathscr{C}=\mathscr{C}_{\mathrm{irr}}=\mathscr{C}_{h}$,

satisfy: (iii) $\Leftrightarrow$ (iv) $\Rightarrow$ (v).

Proof. (i): Let $\phi:[J \cdot R+c \cdot R]_{1} \rightarrow\left[R_{0}\right]_{1} \cong[R / c]_{1}$ be the restrictions map of the natural linear map $[R]_{1} \rightarrow[R / c]_{1} \cdot \phi$ is a linear map with $\operatorname{ker} \phi=c$ and $\operatorname{im} \phi=\left[\sum_{i=1}^{r} \alpha_{i}\right]_{1}$. By a well-known theorem of linear algebra we get

$$
\begin{aligned}
V(1, J \cdot R+c \cdot R) & =\operatorname{dim}_{K}(\operatorname{ker} \phi)+\operatorname{dim}_{K}(\operatorname{Im} \phi) \\
& =V(1, c \cdot R)+V\left(1, \sum_{i=1}^{r} \alpha_{i}\right),
\end{aligned}
$$

and therefore

$$
\begin{aligned}
V(1, J \cdot R \cap c \cdot R) & =V(1, J \cdot R)+V(1, c \cdot R)-V(1, J \cdot R+c \cdot R) \\
& =\sum_{i=1}^{r} V\left(1, \alpha_{i}\right)+V(1, c \cdot R)-V(1, c \cdot R)+V\left(1, \sum_{i=1}^{r} \alpha_{i}\right) \\
& =\sum_{i=1}^{r} V\left(1, \alpha_{i}\right)-V\left(1, \sum_{i=1}^{r} \alpha_{i}\right) .
\end{aligned}
$$

(ii) and the above relations between (iii)-(v): First we need some calculations.

$$
\begin{aligned}
V(1, J \cdot R+c \cdot R) & =V(1, J \cdot R)+V(1, c \cdot R)-V(1 . J \cdot R \cap c \cdot R) \\
& =V(1, J \cdot R)+m+1-V(1, J \cdot R \cap c \cdot R)
\end{aligned}
$$

Further, we get 


$$
\begin{gathered}
V\left(1,\left(J, l_{0}, \ldots, l_{m-e}\right) \cdot \bar{R}\right)=V\left(1,\left(J, l_{0}, \ldots, l_{m-e-1}\right) \cdot \bar{R}\right) \\
+V\left(1,\left(l_{m-e}\right) \cdot \bar{R}\right)-V\left(1,\left(J, l_{0}, \ldots, l_{m-e-1}\right) \cdot \bar{R} \cap\left(l_{m-e}\right) \cdot \bar{R}\right) \\
=V\left(1,\left(J, l_{0}, \ldots, l_{m-e-1}\right) \cdot \bar{R}\right)+1, \text { because } \\
V\left(1,\left(J, l_{0}, \ldots, l_{m-e-1}\right) \cdot \bar{R} \cap\left(l_{m-e}\right) \cdot \bar{R}\right)=V\left(0,\left(J, l_{0}, \ldots, l_{m-e-1}\right) \cdot \bar{R}:\left(\left(l_{m-e}\right) \cdot \bar{R}\right)\right)=0 .
\end{gathered}
$$

(The latter relation follows from the intersection algorithm, because $l_{m-e} \notin\left(J, l_{0}, \ldots, l_{m-e-1}\right) \cdot \bar{R}$.) By induction we obtain:

$$
V\left(1,\left(J, l_{0}, \ldots, l_{m-e}\right) \cdot \bar{R}\right)=V(1, J \cdot R)+m-e+1 .
$$

Proof of (ii): By (i) we have to show that

$$
e \geqq V(1, J \cdot R \cap c \cdot R) .
$$

We get this directly from

$$
V(1, J \cdot R+c \cdot R) \geqq V\left(1,\left(J, l_{0}, \ldots, l_{m-e}\right) \cdot \bar{R}\right)
$$

and the calculations at the beginning of the proof. The equality in (3) is equivalent to

$$
c \cdot \bar{R} \subseteq\left(J, l_{0}, \ldots, l_{m-e}\right) \cdot \bar{R},
$$

but this is the same as (iv). So we have shown the equivalence of (iii) and (iv). (v) follows directly from (iv), because we get $\mathscr{C}=\mathscr{C}_{h}$ and $j\left(X_{1}, \ldots, X_{r} ; C\right)=k\left(X_{1}, \ldots, X_{r} ; C\right)$ for all $C \in \mathscr{C}_{h}=\mathscr{C}$ from the intersection algorithm.

For the first part of conclusion (v) $\left(\mathscr{C}=\mathscr{C}_{h}\right)$ we need (iii) only for all the reduced schemes which appear in the decomposition into irreducible components given by Lemma 1.3, but using the original $e=e\left(X_{1}, \ldots, X_{r}\right)$ instead of the $e$ of the reduced schemes (see also [13]):

Corollary 2.3. Let $X_{1}, \ldots, X_{r}$ be pure dimensional subschemes of $\mathbb{P}_{\mathbf{K}}^{n}$. Let $V_{i j}$, for $1 \leqq j \leqq s_{i}, 1 \leqq i \leqq r$, be the subschemes which are defined according to Lemma 1.3. If

$$
e\left(X_{1}, \ldots, X_{r}\right)=\operatorname{rg}\left(V_{1 j_{1}}, \ldots, V_{r j_{r}}\right) \text { for all } 1 \leqq j_{i} \leqq s_{i}, 1 \leqq i \leqq r,
$$

then $\mathscr{C}\left(X_{1}, \ldots, X_{r}\right)=\mathscr{C}_{h}\left(X_{1}, \ldots, X_{r}\right)$.

Proof. We have

$$
e\left(X_{1}, \ldots, X_{r}\right) \geqq e\left(V_{1 j_{1}}, \ldots, V_{r_{j}}\right) \geqq \operatorname{rg}\left(V_{1 j_{1}}, \ldots, V_{r j_{r}}\right) .
$$


Therefore we get by (\#) $e\left(V_{1 j_{1}}, \ldots, V_{r j_{r}}\right)=\operatorname{rg}\left(V_{1 j_{1}}, \ldots, V_{r j_{r}}\right)$. By Lemma 1.3 and Proposition 2.2 (iii) $\Rightarrow$ (v), it follows that

$$
\mathscr{C}\left(X_{1}, \ldots, X_{r}\right)=\bigcup \mathscr{C}_{h}\left(V_{1 j_{1}}, \ldots, V_{r j_{r}}\right)
$$

Also, by $((\#))$, we get $e\left(X_{1}, \ldots, X_{r}\right)=e\left(V_{1 j_{1}}, \ldots, V_{r j_{r}}\right)$ for all $1 \leqq j_{i} \leqq s_{i}, 1 \leqq i \leqq r$, and we obtain our assertion.

Example (Example 2 from [1]). This example shows that (iii) $\Rightarrow(v)$ is not reversible. Let $X$ and $Y$ be defined by the following ideals:

$$
I(X):=\left(x_{0}^{2}, x_{0} \cdot x_{1}, x_{1}^{2}, x_{0} \cdot x_{3}-x_{1} \cdot x_{2}\right) \text { and } I(Y):=\left(x_{1}, x_{3}\right) .
$$

By Corollary 2.3 we get $\mathscr{C}=\{C\}$, with the intersection point $C$ of $X \cap Y$ which is defined by $x_{0}=x_{1}=x_{3}=0$, and we have

$$
\operatorname{deg} X \cdot \operatorname{deg} Y=k(X, Y ; C) \cdot \operatorname{deg} C=2,
$$

but $\operatorname{rg}(X, Y)=0$ and $e(X, Y)=1$.

Proposition 2.4. Let $X_{1}, \ldots, X_{r}$ be reduced subschemes of $\mathbb{P}_{K}^{n}$ which are connected in codimension one, and let

$$
\mathrm{rg}:=\operatorname{rg}\left(X_{1}, \ldots, X_{r}\right), e:=e\left(X_{1}, \ldots, X_{r}\right) .
$$

Then

$$
\prod_{i=1}^{r} \operatorname{deg} X_{i} \geqq \sum_{C \in \mathscr{E}_{1 \mathrm{rr}}} k\left(X_{1}, \ldots, X_{r} ; C\right) \cdot \operatorname{deg} C+(e-\mathrm{rg}) .
$$

Proof. The proof of Lemma 1.2 in [1] needs the reducedness and the connectedness in codimension 1 of the join-variety $\mathscr{J}$ in $\mathbb{P}_{K}^{n}$ and the $\left({ }^{*}\right)$-condition. The $\left({ }^{*}\right)$-condition is especially used to ensure that the defining ideal $J$ of the join-variety $\mathscr{J}$ and the diagonal ideal $c$ contain no common linear forms (Lemma 2.1 and Proposition 2.2 (i)). The first two of the properties of the join-variety $\mathscr{J}$ mentioned above follow from the same properties for $X_{1}, \ldots, X_{r}$ (see [1]).

We consider the natural ring epimorphism

$$
\phi: \bar{R} \rightarrow \bar{R}^{\prime}:=\bar{R} /\left([J \cdot \bar{R} \cap c \cdot \bar{R}]_{1}\right) .
$$

Let $J^{\prime}$ and $c^{\prime}$ be the images of $J \cdot \bar{R}$ and $c \cdot \bar{R}$ respectively in $\bar{R}^{\prime}$. In the projective space $\mathbb{P}_{\bar{K}}^{m-r g}$ which corresponds to $\bar{R}^{\prime}$, the above assumptions are satisfied. The lengths of the prime ideals which appear are preserved by the surjectivity of $\phi$. So we obtain 


$$
e\left(\mathscr{J}^{\prime}, \Delta^{\prime}\right)=e(\mathscr{J}, \Delta)-\mathrm{rg}=e\left(X_{1}, \ldots, X_{r}\right)-\mathrm{rg}
$$

Corollary 2.5. Let $X_{1}, \ldots, X_{r}$ be pure dimensional subschemes of $\mathbb{P}_{\mathrm{K}}^{n}$. Furthermore, let $X_{i}=\bigcup_{j=1}^{s_{i}} X_{i j}$ be the decomposition of $X_{i}$ into irreducible components, let $V_{i j}=\left(X_{i j}\right)_{\mathrm{red}}$ and let $l_{i j}$ be the length of $\mathcal{O}_{X_{i j}, V_{i j}}$, for $j=1, \ldots, s_{i}$ and $i=1, \ldots, r$, in accordance with Lemma 1.3. Then

$$
\prod_{i=1}^{r} \operatorname{deg} X_{i} \geqq \sum_{i} p_{i} \cdot \sum_{C \in \mathscr{C}_{i}} k\left(V_{1 j_{1}}, \ldots, V_{r j_{r}} ; C\right) \cdot \operatorname{deg} C+\sum_{i} p_{i} \cdot\left(e_{i}-\mathrm{rg}_{1}\right)
$$

where the sum is taken over all $l=\left(j_{1}, \ldots, j_{r}\right)$, with $1 \leqq j_{i} \leqq s_{i}$ and $1 \leqq i \leqq r . \mathscr{C}_{1}, p_{l}, e_{1}$ and $\mathrm{rg}_{\text {, }}$ are defined as follows:

$$
\begin{gathered}
\mathscr{C}_{i}:=\mathscr{C}_{\mathrm{irr}}\left(V_{1 j_{1}}, \ldots, V_{r j_{r}}\right), \\
p_{i}:=l_{1 j_{1}} \cdot \ldots \cdot l_{r j_{r}}, \\
e_{i}:=e\left(V_{1 j_{1}}, \ldots, V_{r j_{r}}\right) \text { and }\left(r g_{i}:=\operatorname{rg}\left(V_{1 j_{1}}, \ldots, V_{r j_{r}}\right) .\right.
\end{gathered}
$$

$\left(\right.$ We set $k\left(V_{1 j_{1}}, \ldots, V_{r j r} ; C\right)$ equal to zero, if $\left.C \notin \mathscr{C}_{\imath}\right)$

Proof. The assumptions of Proposition 2.4 are satisfied for $V_{1 j_{1}}, \ldots, V_{r j_{r}}$. Therefore we get

$$
\prod_{i=1}^{r} \operatorname{deg} V_{i j_{i}} \geqq \sum_{C \in \mathscr{C}_{i}} k\left(V_{1 j_{1}}, \ldots V_{r j_{r}} ; C\right) \cdot \operatorname{deg} C+\left(e_{i}-\mathrm{rg}_{1}\right)
$$

$\prod_{i=1}^{r} \operatorname{deg} X_{i}=\sum_{1} p_{i} \cdot \prod_{i=1}^{r} \operatorname{deg} V_{i j_{i}}$ and Lemma 1.3 proves the corollary.

Theorem 2.6 Let us take the same assumptions and notation as in Corollary 2.5. Furthermore let $\mathscr{C}_{i}^{h}:=\mathscr{C}_{h}\left(V_{1 j_{1}}, \ldots, V_{r j_{r}}\right)$. Then the following conditions are equivalent:

(i) $\prod_{i=1}^{r} \operatorname{deg} X_{i} \leqq \sum_{i} p_{i} \cdot \sum_{C_{\epsilon} \mathscr{C}_{1}^{h}} k\left(V_{1 j_{1}}, \ldots, V_{r j_{r}} ; C\right) \cdot \operatorname{deg} C$,

(ii) $\prod_{i=1}^{r} \operatorname{deg} X_{i} \leqq \sum_{1} p_{i} \cdot \sum_{c_{\in} \mathscr{C}_{1}} k\left(V_{1 j_{1}}, \ldots, V_{r j} ; C\right) \cdot \operatorname{deg} C$,

(iii) $e_{i}=\mathrm{rg}_{1}$ for all $l=\left(j_{1}, \ldots, j_{r}\right)$ with $1 \leqq j_{i} \leqq s_{i}$ and $1 \leqq i \leqq r$.

Proof. (i) $\Rightarrow$ (ii) is trivial.

(ii) $\Rightarrow$ (iii) follows from Corollary 2.5 .

(iii) $\Rightarrow$ (i): By proposition 2.2 (iii) $\Rightarrow$ (v) we obtain

$$
\prod_{i=1}^{r} \operatorname{deg} V_{i j_{1}}=\sum_{C \in \mathscr{\mho}_{i}^{h}} k\left(V_{1 j_{1}}, \ldots, V_{r j_{r}} ; C\right) \cdot \operatorname{deg} C
$$


(i) follows in the same way as in the proof of Corollary 2.5. Theorem 2.7 shows that the Theorem in [13] cannot be completely extended to the case $\mathscr{C}=\mathscr{C}_{\mathrm{irr}}$, and the subsequent example shows the relevance of this case.

Theorem 2.7. Let $X_{1}, \ldots, X_{r}$ be pure dimensional schemes of $\mathbb{P}_{K}^{n}$. With the same assumptions and notation as in Corollary 2.5 we get for the following conditions

(i) $\prod_{i=1}^{r} \operatorname{deg} X_{i}=\sum_{c \in \mathscr{C}_{i r r}} k\left(X_{1}, \ldots, X_{r} ; C\right) \cdot \operatorname{deg} C$,

(ii) $\prod_{i=1}^{r} \operatorname{deg} X_{i} \leqq \sum_{c \in \mathscr{C}_{\mathrm{irr}}} k\left(X_{1}, \ldots, X_{r} ; C\right) \cdot \operatorname{deg} C$,

(iii) $e_{1}=\operatorname{rg}_{1}$ for all $l=\left(j_{1}, \ldots, j_{r}\right)$ with $1 \leqq j_{i} \leqq s_{i}$ and $1 \leqq i \leqq r$,

(iv) $\prod_{i=1} \operatorname{deg} X_{i}=\sum_{C \in \mathscr{C}_{i r r}} j\left(X_{1}, \ldots, X_{r} ; C\right) \cdot \operatorname{deg} C$,

(i) $\Leftrightarrow$ (ii) $\Rightarrow$ (iii), (iv).

Furthermore, the following conclusions are in general false: (iii) $\Rightarrow$ (i) or (iv), (iv) $\Rightarrow$ (i) or (iii).

Proof. (i) $\Rightarrow$ (ii) is trivial.

(ii) $\Rightarrow$ (i), (iv) resp.:

We look at following chain of inequalities:

$$
\begin{gathered}
\sum_{C \in \mathscr{C}_{i \mathrm{ir}}} k\left(X_{1}, \ldots, X_{r} ; C\right) \cdot \operatorname{deg} C \geqq \prod_{i=1}^{r} \operatorname{deg} X_{i} \\
\geqq \sum_{C \in \mathscr{C}_{i \mathrm{irr}}} j\left(X_{1}, \ldots, X_{r} ; C\right) \cdot \operatorname{deg} C \\
\geqq \sum_{C \in \mathscr{Q}_{i \mathrm{ir}}} k\left(X_{1}, \ldots, X_{r} ; C\right) \cdot \operatorname{deg} C .
\end{gathered}
$$

Therefore we get equality at every point of the chain, and so we obtain (i) and (iv).

(ii) $\Rightarrow$ (iii): Because of (i) and (iv) we get $\mathscr{C}=\mathscr{C}_{\text {irr }}$ and for every $C \in \mathscr{C}$

$$
k\left(X_{1}, \ldots, X_{r} ; C\right)=j\left(X_{1}, \ldots, X_{r} ; C\right) .
$$

By Lemmas 3 and 4 of [13] we get the following results:

(1) $\mathscr{C}\left(V_{1 j_{1}}, \ldots, V_{r j_{r}}\right)=\mathscr{C}_{i r r}\left(V_{1 j_{1}}, \ldots, V_{r j_{r}}\right)$ for all $l$,

(2) $k\left(V_{1 j_{1}}, \ldots, V_{r j_{r}} ; C\right)=j\left(V_{1 j_{1}}, \ldots, V_{r j_{r}} ; C\right)$ for all $l$.

By Proposition 2.4 and consequences 1 and 2, we deduce the following chain of inequalities:

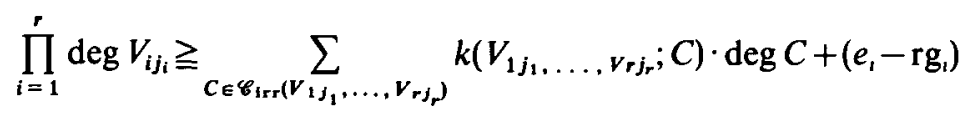




$$
\begin{aligned}
& =\sum_{C \in \mathscr{C}_{i \mathrm{rr}}\left(V_{1 j_{1}}, \ldots, V_{r j_{r}}\right)} j\left(V_{1 j_{1}, \ldots, V_{r j_{r}}} ; C\right) \cdot \operatorname{deg} C+\left(e_{1}-\mathrm{rg}_{\imath}\right) \\
& =\sum_{C \in \mathscr{G}\left(V_{1}, \ldots \ldots V_{r_{r}}\right)} j\left(V_{1 j_{1}} \ldots \ldots V_{r j} ; C\right) \cdot \operatorname{deg} C+\left(e_{1}-\mathrm{rg}_{1}\right) \\
& =\prod_{i=1}^{r} \operatorname{deg} V_{i j_{i}}+\left(e_{t}-\mathrm{rg}_{1}\right)
\end{aligned}
$$

for all $\imath=\left(j_{1}, \ldots j_{r}\right)$ with $1 \leqq j_{i} \leqq s_{i}$ and $1 \leqq i \leqq r$. Therefore we obtain (iii).

Counterexamples for (iv) $\Rightarrow$ (ii) or (iii) and (iii) $\Rightarrow$ (ii) are contained in [13]. Therefore we need for the proof of the theorem a counterexample to (iii) $\Rightarrow$ (iv):

We set $I(X)=\left(x_{0}^{2} \cdot x_{1}\right)$ and $I(Y)=\left(x_{0} \cdot x_{1}^{2}\right)$ in $K\left[x_{0}, x_{1}, x_{2}\right]$. Following the method of decomposition we get

$$
I\left(X_{1}\right)=\left(x_{0}^{2}\right), I\left(X_{2}\right)=\left(x_{1}\right) \text { and } I\left(Y_{1}\right)=\left(x_{0}\right), I\left(Y_{2}\right)=\left(x_{1}^{2}\right)
$$

So it follows that

$$
e_{i j}=\left\{\begin{array}{ll}
1 & \text { for } i=j \\
0 & \text { otherwise }
\end{array}\right\}=r_{i j} \quad \text { for } i, j=1,2
$$

Therefore (iii) holds, and by an easy computation using Lemma 1.3 we know that (iv) is false.

Example. Let $X$ and $Y$ be subschemes of $\mathbb{P}_{K}^{5}$ with defining ideals

$$
I(X)=\left(x_{0}, x_{1}, x_{2}\right) \cap\left(x_{3}, x_{4}, x_{5}\right) \text { and } I(Y)=\left(x_{1}, x_{2}, x_{3}\right) \text {. }
$$

$X$ and $Y$ are reduced, $e(X, Y)=2, \operatorname{rg}(X, Y)=0$ and

$$
I(X)+I(Y)=\left(x_{0}, x_{1}, x_{2}, x_{3}\right) \cap\left(x_{1}, x_{2}, x_{3}, x_{4}, x_{5}\right) .
$$

By Lemma 1.3 we get

$$
\mathscr{C}=\left\{C_{1}: x_{0}=x_{1}=x_{2}=x_{3}=0, C_{2}: x_{1}=x_{2}=x_{3}=x_{4}=x_{5}=0\right\}
$$

and

$$
\operatorname{deg} X \cdot \operatorname{deg} Y=2 \cdot 1=1+1=\operatorname{deg} C_{1}+\operatorname{deg} C_{2} .
$$

We note that this example shows once more that the formula of Lazarsfeld (see [3, Ex. 12.3.5]) cannot be extended to reduced schemes.

For the following key lemma which we use to generlaize Proposition 2.4 to schemes which are connected in codimension one (see Theorem 2.9) we need some notation. Let 
$\alpha$ be a homogenous ideal in $K\left[x_{0}, \ldots, x_{n}\right] . H(t, \alpha)$ denotes its Hilbert function. This function is for large $t$ a polynomial in $t$ and this polynomial can be written in the following form:

$$
H(t, \alpha)=h_{0}(\alpha) \cdot\left(\begin{array}{l}
t \\
d
\end{array}\right)+h_{1}(\alpha) \cdot\left(\begin{array}{c}
t-1 \\
d
\end{array}\right)+\cdots+h_{d}(\alpha)
$$

where $d=$ Krull-dim $\alpha-1$ and the so-called Hilbert coefficients $h_{0}(\alpha)>0, h_{1}(\alpha), \ldots, h_{d}(\alpha)$ are integers. (For some properties of the Hilbert function and its coefficients see [6, (1.41)] or [12, Ch. I C].)

Lemma 2.8. Let $X_{1}, \ldots, X_{r}$ be pure dimensional subschemes of $\mathbb{P}_{K}^{n}$. Then for all components $C$ of the collection $\mathscr{C}\left(X_{1}, \ldots, X_{r}\right)$

$$
\begin{aligned}
j\left(\left(X_{1}\right)_{\mathrm{red}}, \ldots,\left(X_{r}\right)_{\mathrm{red}} ; C\right) & -k\left(\left(X_{1}\right)_{\mathrm{red}}, \ldots,\left(X_{r}\right)_{\mathrm{red}} ; C\right) \\
& \leqq j\left(X_{1}, \ldots, X_{r} ; C\right)-k\left(X_{1}, \ldots, X_{r} ; C\right)
\end{aligned}
$$

Proof. By Lemma 1.3 (i) $\mathscr{C}\left(\left(X_{1}\right)_{\text {red }}, \ldots,\left(X_{\text {r }}\right)_{\text {red }}\right)=\mathscr{C}\left(X_{1}, \ldots, X_{r}\right)$. So the intersection numbers $j\left(\left(X_{1}\right)_{\text {red }}, \ldots,\left(X_{r}\right)_{\text {red }} ; C\right)$ and $k\left(\left(X_{1}\right)_{\text {red }}, \ldots,\left(X_{\mathrm{r}}\right)_{\text {red }} ; C\right)$ are defined. Multiplying both sides of the above inequality by $h_{0}(p+c)$, where $p$ is the defining ideal of $C$ in $\bar{K}\left[x_{0}, \ldots, x_{n}\right]$ and $c$ is the diagonal ideal of $\bar{R}$, we get the equivalent inequality:

$$
h_{0}(q)-h_{0}\left(q_{\mathrm{red}}\right) \geqq h_{0}(q+c)-h_{0}\left(q_{\mathrm{red}}+c\right),
$$

where $q$ (resp. $q_{\text {red }}$ ) is the primary ideal corresponding to $p+c$ which is constructed in the intersection algorithm for $X_{1}, \ldots, X_{r}$ (resp. for $\left.\left(X_{1}\right)_{\text {red }}, \ldots,\left(X_{r}\right)_{\text {red }}\right)$.

Let $\operatorname{dim} C=\operatorname{dim}\left(X_{1} \cap \cdots \cap X_{r}\right)-i$. Then we know by the definitions of Section 1 that

$$
\begin{gathered}
q+c=\left(q, l_{m-e+i+1}, \ldots, l_{m}\right), \text { and } \\
q_{\mathrm{red}}+c=\left(q_{\mathrm{red}}, l_{m-e+i+1}, \ldots, l_{m}\right), \text { respectively. }
\end{gathered}
$$

We define for $m-e+i<j \leqq m$

$$
\begin{aligned}
& q^{(j)}:=\left(q, l_{m-e+i+1}, \ldots, l_{j-1}\right) \text { and } \\
& q_{\mathrm{red}}^{(j)}:=\left(q_{\mathrm{red}}, l_{m-e+i+1}, \ldots, l_{j-1}\right) .
\end{aligned}
$$

Claim. $q^{(j)} \subseteq q_{\mathrm{red}}^{(j)}$ for all $m-e+i<j \leqq m$.

For the proof of the claim it is sufficient to show that

$$
q \subseteq q_{\mathrm{red}}
$$

For two ideals $\alpha$ and $b$ of a Noetherian ring $R$ of the same dimension $d$ we note that an 
inclusion $\alpha \subseteq b$ is transferred to inclusions of such $d$-dimensional primary components of $\alpha$ and $b$ belonging to the prime ideals of $b$. This one can see by localization.

Let $\alpha_{1}, \ldots, \alpha_{r}$ be the defining ideals in $K\left[x_{0}, \ldots, x_{n}\right]$ of $X_{1}, \ldots, X_{r}$ respectively. Then it is clear that

$$
J:=\alpha_{1}^{\prime}+\cdots+\alpha_{r}^{\prime} \subseteq \operatorname{Rad}\left(\alpha_{1}\right)^{\prime}+\cdots+\operatorname{Rad}\left(\alpha_{r}\right)^{\prime}=: J_{\mathrm{red}}
$$

With the notation of Section 1 we get from the above remark that

$$
\begin{array}{ll}
U\left((J \cdot \bar{R})_{\mu} \subseteq U\left(\left(J_{\text {red }} \cdot \bar{R}\right)_{\mu}\right)\right. & \text { for all } \mu=-1, \ldots, m-e \text { and } \\
\mathfrak{U}_{v} \subseteq \mathfrak{U}_{v}^{\text {red }} & \text { for all } \nu,
\end{array}
$$

where the $\mathfrak{U}_{v}^{\text {red }}$ is constructed in the intersection algorithm by using $J_{\text {red }}$ instead of $J$. Now the claim follows from the last inclusion for $v=i$ by localization.

Now we complete the proof of Lemma 2.8. We get (cf. $[12,(1.36)]$ )

$$
\begin{gathered}
h_{0}\left(q^{(j)}, l_{j}\right)=h_{0}\left(q^{(j)}\right)-h_{0}\left(q^{(j)}: l_{j}\right) \text { and } \\
h_{0}\left(q_{\mathrm{red}}^{(j)}, l_{j}\right)=h_{0}\left(q_{\mathrm{red}}^{(j)}\right)-h_{0}\left(q_{\mathrm{red}}^{(j)}: l_{j}\right), \text { respectively }
\end{gathered}
$$

Therefore

$$
\begin{aligned}
& h_{0}\left(q^{(j+1)}\right)-h_{0}\left(q_{\mathrm{red}}^{(j+1)}\right)=h_{0}\left(q^{(j)}, l_{j}\right)-h_{0}\left(q_{\mathrm{red}}^{(j)}, l_{j}\right) \\
& =h_{0}\left(q^{(j)}\right)-h_{0}\left(q_{\mathrm{red}}^{(j)}\right)-\left(h_{0}\left(q^{(j)}: l_{j}\right)-h_{0}\left(q_{\mathrm{red}}^{(j)}: l_{j}\right)\right)
\end{aligned}
$$

where we set those $h_{0}$ of $q^{(j)}: l_{j}$ or of $q_{\mathrm{red}}^{(j)}: l_{j}$ equal to zero, if their dimensions are lower than $\operatorname{dim}(p+c)$.

By the claim we get $h_{0}\left(q^{(j)}: l_{j}\right) \geqq h_{0}\left(q_{\text {red }}^{(j)}: l_{j}\right)$, and so

$$
h_{0}\left(q^{(j+1)}\right)-h_{0}\left(q_{\text {red }}^{(j+1)}\right) \leqq h_{0}\left(q^{(j)}\right)-h_{0}\left(q_{\text {red }}^{(j)}\right)
$$

This gives us a chain of inequalities and proves the lemma.

Theorem 2.9. Let $X_{1}, \ldots, X_{r}$ be pure dimensional subschemes of $\mathbb{P}_{K}^{n}$ which are connected in codimension one. Then

$$
\prod_{i=1}^{r} \operatorname{deg} X_{i} \geqq \sum_{C \in \mathcal{Q}_{\mathrm{irr}}} k\left(X_{1}, \ldots, X_{r} ; C\right) \cdot \operatorname{deg} C+\left(e-\mathrm{rg}_{\mathrm{red}}\right)
$$

Proof. For brevity we set 


$$
k_{C}:=k\left(X_{1}, \ldots, X_{r} ; C\right), k_{C}^{\text {red }}:=k\left(\left(X_{1}\right)_{\text {red }}, \ldots,\left(X_{r}\right)_{\text {red }} ; C\right)
$$

and

$$
j_{C}:=j\left(X_{1}, \ldots, X_{r} ; C\right), j_{C}^{\mathrm{red}}:=j\left(\left(X_{1}\right)_{\mathrm{red}}, \ldots,\left(X_{r}\right)_{\mathrm{red}} ; C\right) .
$$

By Proposition 2.4

$$
\prod_{i=1}^{r} \operatorname{deg}\left(X_{i}\right)_{\mathrm{red}} \geqq \sum_{C \in \mathscr{\varphi}_{\mathrm{irr}}} k_{C}^{\mathrm{red}} \cdot \operatorname{deg} C+\left(e-\mathrm{rg}_{\mathrm{red}}\right) .
$$

Rearranging this inequality we get by Lemma 1.1 .

$$
\sum_{C \in \mathscr{E}_{\mathrm{irr}}}\left(j_{C}^{\mathrm{red}}-k_{C}^{\mathrm{red}}\right) \cdot \operatorname{deg} C+\sum_{C \in \mathscr{\mathscr { E }} \backslash \mathscr{Q}_{\mathrm{irr}}} j_{C}^{\mathrm{red}} \cdot \operatorname{deg} C \geqq e-\mathrm{rg}_{\mathrm{red}} \cdot
$$

By Lemma 1.3 we get $j_{c} \geqq j_{C}^{\text {red }}$; therefore we obtain the assertion, by Lemma 2.8 .

Corollary 2.10. Let $X_{1}, \ldots, X_{r}$ be as in Theorem 2.9. Then the following conditions are equivalent:

(i) $\prod_{i=1}^{r} \operatorname{deg} X_{i} \leqq \sum_{C \in \mathscr{E}_{i r r}} k\left(X_{1}, \ldots, X_{r} ; C\right) \cdot \operatorname{deg} C$,

(ii) $\prod_{i=1}^{r} \operatorname{deg} X_{i} \leqq \sum_{c \in \mathscr{\ell}_{n}} k\left(X_{1}, \ldots, X_{r} ; C\right) \cdot \operatorname{deg} C$.

Proof. (ii) $\Rightarrow$ (i) obvious.

(i) $\Rightarrow$ (ii): By Theorem 2.9 we get $e=\mathrm{rg}_{\mathrm{red}}$, and by Proposition 2.2 (v)

$$
\prod_{i=1}^{r} \operatorname{deg}\left(X_{i}\right)_{\mathrm{red}}=\sum_{C \in \mathscr{C}_{h}} k\left(\left(X_{1}\right)_{\mathrm{red}}, \ldots,\left(X_{r}\right)_{\mathrm{red}} ; C\right) \cdot \operatorname{deg} C .
$$

In particular this shows $\mathscr{C}=\mathscr{C}_{\text {irr }}=\mathscr{C}_{h}$.

\section{The Cohen-Macaulay case}

In this section we apply Theorem 2.9 to the case of Cohen-Macaulay schemes and we discuss particularly the so-called general Bézout's Theorem. This theorem is a generalization of the case of the intersection of hypersurfaces. This is important, because hypersurfaces are in general neither reduced nor irreducible. First we transfer Theorem 2.9 to this case.

Proposition 3.1. Let $X_{1}, \ldots, X_{r}$ be subschemes of $\mathbb{P}_{K}^{n}$ which are connected in codimension one. Let the local Rings $\mathcal{O}_{x_{i}, \mathrm{C}}$ be Cohen-Macaulay for all $C \in \mathscr{C}_{\text {irr }}$ and all $i=1, \ldots, r$. Then 


$$
\prod_{i=1}^{r} \operatorname{deg} X_{i} \geqq \operatorname{degg}\left(X_{1} \cap \cdots \cap X_{\mathrm{r}}\right)+\left(e-\mathrm{rg}_{\mathrm{red}}\right)
$$

Proof. By the Cohen-Macaulay property and [1, Proposition 3.8]

$$
k\left(X_{1}, \ldots, X_{r} ; C\right)=l\left(X_{1}, \ldots, X_{r} ; C\right)
$$

for all $C \in \mathscr{C}_{\mathrm{irr}}$ (Here $l\left(X_{1}, \ldots, X_{r} ; C\right):=$ length of $\mathcal{O}_{X_{1} \cap \ldots \cap X_{r}, c}$.) Our assertion follows from Theorem 2.9 .

We deduce a new proof of [2, Proposition 3.12].

Corollary 3.2. Let $X_{1}, \ldots, X_{r}$ and the notation be as in Proposition 3.1. If $\mathrm{rg}_{\mathrm{red}}=0$, then the following conditions are equivalent:

(i) $\operatorname{deg}\left(X_{1} \cap \cdots \cap X_{r}\right) \geqq \prod_{i=1}^{r} \operatorname{deg} X_{i}$,

(ii) $\operatorname{deg}\left(X_{1} \cap \cdots \cap X_{r}\right) \geqq \prod_{i=1}^{r} \operatorname{deg} X_{i}$,

(iii) $e\left(X_{1}, \ldots, X_{r}\right)=0$.

Proof. (i) $\Rightarrow$ (ii) is trivial.

(ii) $\Rightarrow$ (ii) follows from Proposition 3.1 and $\mathrm{rg}_{\text {red }}=0$.

(iii) $\Rightarrow$ (i): Since $e=0$ we get the following consequences:

(1) $\mathscr{C}=\mathscr{C}_{h}$ and

(2) $j\left(X_{1}, \ldots, X_{r} ; C\right)=k\left(X_{1}, \ldots, X_{r} ; C\right)$ for all $C \in \mathscr{C}$.

From the Cohen-Macaulay property it follows as in Proposition 3.1 that

$$
k\left(X_{1}, \ldots, X_{r} ; C\right)=l\left(X_{1}, \ldots, X_{r} ; C\right) .
$$

Corollary 3.3. Let $X_{1}, \ldots, X_{r}$ be arithmetically Cohen-Macaulay schemes of $\mathbb{P}_{K}^{n}$ of dimension $\geqq 1$. Then

$$
\prod_{i=1}^{r} \operatorname{deg} X_{i} \geqq \operatorname{deg}\left(X_{1} \cap \cdots \cap X_{r}\right)+\left(e-\mathrm{rg}_{\mathrm{red}}\right)
$$

Proof. By Theorem 5 of [10] we get that the $X_{i}$ are connected in codimension one. Proposition 3.1. completes the proof.

Since $[10]$ is sometimes not readily available we want to give here a short sketch of a proof of this fact under our assumptions.

Claim. If $V$ is an arithmetically Cohen-Macaulay scheme of $\mathbb{P}_{K}^{n}$ of dimension $\geqq 1$. Then $V$ is connected in codimension one.

Proof. Let $V$ be defined by the homogeneous ideal $\alpha$ of $R:=K\left[x_{0}, \ldots, x_{n}\right\}$. By our assumption we get that all the local rings $R_{p} / \alpha \cdot R_{p}$ for every prime ideals $p$ with 
$\alpha \subseteq p \subseteq R$ satisfy the condition $S_{2}$ by Serre. By [7, Corollary 2.4] and Remark 1.3.2 we get our assertion if we know that $V$ is connected. For this we consider the affine cone $X$ over $V . X$ is a connected noetherian topological space. Let $X$ be the vertex of $X$. We set $Y:=\{x\}$. By [7, Theorem 2.2], we get $X \backslash\{x\}$ is connected, since depth $\mathcal{O}_{X, x} \geqq 2$ because of the Cohen-Macaulay property. Therefore $V$ is connected.

For arithmetically Cohen-Macaulay schemes we can improve Corollary 3.2 to the following general Bézout's theorem.

Theorem 3.4. Let $X_{1}, \ldots, X_{r}$ be arithmetically Cohen-Macaulay schemes of $\mathbb{P}_{K}^{n}$. Then the following conditions are equivalent.

(i) $\tilde{\operatorname{deg}}\left(X_{1} \cap \cdots \cap X_{r}\right) \geqq \prod_{i=1}^{r} \operatorname{deg} X_{i}$

(ii) $\operatorname{deg}\left(X_{1} \cap \cdots \cap X_{r}\right) \geqq \prod_{i=1}^{r} \operatorname{deg} X_{i}$

(iii) $e\left(X_{1}, \ldots, X_{r}\right)=\operatorname{rg}\left(X_{1}, \ldots, X_{r}\right)$.

Proof. By Corollary 2.10, (i) and (ii) are equivalent.

(ii) $\Rightarrow$ (iii) is Proposition 2 from [13].

(iii) $\Rightarrow$ (ii): By Proposition 2.2, we get that property, $(v)$ in Proposition 2.2 holds, and as in Proportion 3.1 this proves the theorem, using the Cohen-Macaulay property.

Remark. By defining $E\left(X_{1}, \ldots, X_{r}\right):=\mathrm{e}\left(X_{1}, \ldots, X_{r}\right)-\operatorname{rg}\left(X_{1}, \ldots, X_{r}\right)$ we get following "geometric" statement:

Let $X_{1}, \ldots, X_{r}$ be arithmetically Cohen-Macaulay schemes of $\mathbb{P}_{K}^{n}$. Then the following conditions are equivalent.

(i) $\operatorname{deg}\left(X_{1} \cap \cdots \cap X_{r}\right) \geqq \prod_{i=1}^{r} \operatorname{deg} X_{i}$,

(ii) $\operatorname{deg}\left(X_{1} \cap \cdots \cap X_{r}\right) \geqq \prod_{i=1}^{r} \operatorname{deg} X_{i}$,

(iii) $E\left(X_{1}, \ldots, X_{r}\right)=0$.

Now we list some interesting consequences.

Corollary 3.5. Let $X_{1}, \ldots, X_{r}$ be arithmetically Cohen-Macaulay schemes of $\mathbb{P}_{K}^{n}$. If $e \neq \mathrm{rg}$, then

$$
\prod_{i=1}^{r} \operatorname{deg} X_{i} \geqq \operatorname{deg}\left(X_{1} \cap \cdots \cap X_{r}\right)+1
$$

For intersections of hypersurfaces we get:

Corollary 3.6. Let $X_{1}, \ldots, X_{r}$ be hypersurfaces of $\mathbb{P}_{K}^{n}$, which are defined by forms $F_{1}, \ldots, F_{r}$ of degree $d_{1}, \ldots, d_{r}$. Then the following conditions are equivalent.

(i) $h_{0}\left(F_{1}, \ldots, F_{r}\right)=\prod_{i=1}^{r} d_{i}$, 
(ii) $e=$ number of linear forms in the set $\left\{F_{1}, \ldots, F_{r}\right\}$-number of linearly independent linear forms in $\left\{F_{1}, \ldots, F_{r}\right\}$.

For $r=2$ we obtain directly from Theorem 3.4 a result of W. Vogel (Proposition 3 in [13]).

Corollary 3.7. Let $X$ and $Y$ be arithmetically Cohen-Macaulay schemes of $\mathbb{P}_{K}^{n}$, which are defined by the homogeneous ideals $\alpha, b$ of $K\left[x_{0}, \ldots, x_{n}\right]$. Then the following conditions are equivalent.

(i) $\operatorname{deg}(X \cap Y) \geqq \operatorname{deg} X \cdot \operatorname{deg} Y$,

(ii) $\operatorname{deg}(X \cap Y) \geqq \operatorname{deg} X \cdot \operatorname{deg} Y$,

(iii) $e(X, Y)=V(1, \alpha \cap b)$.

Proof. Apply the dimension theorem for vector spaces.

Now we show the result of B. Renschuch and W. Vogel from [9].

Corollary 3.8. Let $X_{1}, \ldots, X_{r}$ be as in Proposition 3.4, where $X_{i}$ is defined by the homogeneous ideals $\alpha_{i} \subseteq K\left[x_{0}, \ldots, x_{n}\right]$ for $i=1, \ldots, r$. If the $\alpha_{i}$ satisfy the $\left(^{*}\right)$-condition, then the following conditions are equivalent.

(i) $\operatorname{deg}\left(X_{1} \cap \cdots \cap X_{r}\right) \geqq \prod_{i=1}^{r} \operatorname{deg} X_{i}$,

(ii) $\operatorname{deg}\left(X_{1} \cap \cdots \cap X_{r}\right) \geqq \prod_{i=1}^{r} \operatorname{deg} X_{i}$,

(iii) $e\left(X_{1}, \ldots, X_{r}\right)=0$.

Proof. This follows from Proposition 2.1 and Theorem 3.4.

Acknowledgement. I would like to thank Prof. W. Vogel and Dr. L. O'Carroll for their help during the preparation of this paper.

\section{REFERENCES}

1. H. Flenner and W. Vogel, Connectivity and its application to improper intersection in ppn, Math. Gottingensis 53 (1988).

2. H. Flenner, L. van Gastel and W. Vogel, Joins and intersections, Math. Ann. 291 (1991), 691-704.

3. W. Fulton, Intersection Theory (Berlin-Heidelberg-New York-Tokyo, Springer 1984).

4. W. Fulton, Letter to W. Vogel dated 14 July, 1990.

5. L. vaN GaSTEL, Excess intersections and a correspondence principle, Invent. Math. 103 (1991), 197-221.

6. W. Grönner, Moderne Algebraische Geometrie. Die Idealtheoretischen Grundlagen (WienInsbruck, Springer, 1949).

7. R. Hartshorne, Complete intersections and connectedness, Amer.J. Math. 84 (1962), 497-508. 
8. L. O'Carroll, On bounding the Stückrad-Vogel multiplicity, Proc. Edinburgh Math. Soc. 34 (1991), 251-257.

9. B. Renschuch and W. Vogel, Perfektheit und die Umkehrung des Bezoutschen Satzes, Math. Nachr. 148 (1990), 313-323.

10. H. Roloff and J. Stückrad, Bemerkungen über Zusammenhangseigenschaften und mengentheoretische Darstellung projektiver algebraischer Mannigfaltigkeiten, Beiträge Algebra Geom. 8 (1979), 125-131.

11. J. StÚckrad and W. Vogel, An algebraic approach to the intersection theory, in The curves seminar at Queen's II, Queen's Papers in Pure and Appl. Math. 61 (1982), 1-32.

12. W. Vogel, Lectures on Results on Bezout's Theorem (Tata Lecture notes, Bombay, 74, Berlin-Heidelberg-New York-Tokyo, Springer 1984).

13. W. Vogel, A converse of Bézout's theorem, Ann. Univ. Ferrara Sez. VII (N.S.), 36 (1990), 85-95.

14. W. Vogel, On multiplicities of non-isolated intersection components, Publ. Res. Inst. Math. Sci. 27 (1992), 845-860.

Martin-Luther-Universität Halle-Wittenberg

FaChBeREICH MathematiK UND INFORMatiK

D 06099 HaLLE/S

Germany 